\title{
Hopf Cyclic Cohomology in Non-symmetric Monoidal Categories
}

\author{
Arash Pourkia \\ ${ }^{1}$ Huron University College, Western University, Canada \\ ${ }^{2}$ American University of Middle East (in affiliation with Purdue University), Kuwait
}

Copyright (C)2015 by authors, all rights reserved. Authors agree that this article remains permanently

open access under the terms of the Creative Commons Attribution License 4.0 International License

\begin{abstract}
First, referring to our previous work, "Hopf cyclic cohomology in braided monoidal categories", we reduce the restriction of the ambient category $\mathcal{C}$ being symmetric. We let $\mathcal{C}$ to be non-symmetric but assume only the restriction, $\psi^{2}=i d$, on the braid map correspond to the Hopf algebra $H$, which is the main player in the theory. We define a family of examples of such desired braided Hopf algebras, $H$, living in the category of anyonic vector spaces. Next, on one hand, we will prove that these anyonic Hopf algebras are the enveloping (Hopf) algebras of particular quantum Lie algebras, which we will construct. On the other hand, we will show that, analogous to the non-super and the super case, the well known relationship between the periodic Hopf cyclic cohomology of an enveloping (super) algebra and the (super) Lie algebra homology also holds for these particular quantum Lie algebras.
\end{abstract}

Keywords Hopf Cyclic Cohomology, Braided Monoidal Categories, Braided Hopf Algebras, Quantum Lie Algebras

\section{Introduction}

In [2, 3, 4], Connes and Moscovici, motivated by transverse index theory for foliations, defined a cohomology theory of cyclic type for Hopf algebras endowed with a modular pair in involution (MPI). This theory was later extended in [7, 8] to the more general case of Hopf cyclic cohomology with coefficients, by introducing the notion of stable anti Yetter-Drinfeld (SAYD) modules.

In [9] we extended all these formalisms of Hopf cyclic cohomology to the context of abelian braided monoidal categories. When the braiding is symmetric we associated a cocyclic object to a braided Hopf algebra endowed with a braided modular pair in involution. When the braiding is non-symmetric we obtained a para-cocyclic object instead of a cocyclic object. To obtain a cocyclic object from a para-cocyclic object one has to work in an appropriate subspace. We also refer the reader to [15], for a quick review of the subject of (braided) Hopf cyclic cohomology, and for more references on the literature.

This paper is organized as follows. In Section 2, in Theorems 2.3 and 2.4, we reduce the restriction of the ambient category $\mathcal{C}$ being symmetric, i.e., $\psi_{A \otimes B} \psi_{B \otimes A}=i d_{B \otimes A}$, for all $A$ and $B$ in $\mathcal{C}$, to a less restrictive condition. We show that even in the non-symmetric case as long as we only have the relation $\psi_{H \otimes H}^{2}=\psi_{H \otimes H} \psi_{H \otimes H}=i d_{H \otimes H}$ we will still obtain a cocyclic object. In the case of Hopf cyclic cohomology with coefficients in an $H$-module $M$ we also need to have the relation $\psi_{H \otimes M} \psi_{M \otimes H}=i d_{M \otimes H}$. In the most general case, for the triple $(H, C, M)$, we also need similar restrictions on $\psi_{H \otimes C}$, $\psi_{M \otimes C}$ and $\psi_{C \otimes C}$. This is because the main players in the theory are $H, M$ and $C$ and, in fact, nowhere in any of the proofs of the relevant theorems in [9] we need more than the above mentioned relations.

In Section 3 we provide a family of examples of such a desired situation where the ambient category $\mathcal{C}$ is not symmetric but for many objects $A$ and $B$ in $\mathcal{C}$ the property $\psi_{A \otimes B} \psi_{B \otimes A}=i d_{B \otimes A}$ holds. The category that we will work in is the category of anyonic vector spaces $[12,14]$. Then, we provide families of examples of anyonic Hopf algebras, $H$, for which $\psi_{H \otimes H}^{2}=i d_{H \otimes H}$, so that our Theorem 2.4 could be applied to these examples.

In Section 4 we construct a family of particular quantum Lie algebras, $\mathfrak{g}$, in the sense of [6] (also with the property $\left.\psi^{2}=i d\right)$, in the same category of anyonic vector spaces. Then, we will show that their universal enveloping algebras, $U(\mathfrak{g})$, are the anyonic Hopf algebras introduced in Section 3.

We should mention that in [6], they have shown, among other things, that the notion of quantum Lie algebras, in the sense of [16], is closely related to braided Lie algebras, in the sense of [13]. To do this, they have provided an axiomatic definition 
of (left) quantum Lie algebras. In this paper, we follow [6] for the definition of a quantum Lie algebra, and [12, 13, 14] for other definitions and notions we need.

In Section 5 we present Theorem 5.1, to compute the (braided) periodic Hopf cyclic cohomology of the anyonic Hopf algebras of Section 3. It states that, analogous to the non-super [2, 3, 4, 5] and the super case [9], the well known relationship between the periodic Hopf cyclic cohomology of an enveloping algebra, $H P_{(\delta, 1)}^{*}(U(\mathfrak{g}))$, and the Lie algebra homologies, $H_{i}\left(\mathfrak{g} ; \mathbb{C}_{\delta}\right)$, holds for those quantum Lie algebras of Section 4.

To keep this note short we have not included preliminaries.

\section{Hopf cyclic theory in non-symmetric categories}

In this section we first present an analogous of Theorem 3.6 in [9], with the restriction of the ambient category being symmetric replaced by a more less restrictive one. We assign a cocyclic object to a braided triple $(H, C, M)$ in an abelian braided monoidal category $\mathcal{C}$ but with the symmetric condition for $\mathcal{C}$ replaced by only restrictions on $\psi_{H \otimes M}, \psi_{H \otimes C}, \psi_{M \otimes C}$, $\psi_{H \otimes H}$ and $\psi_{C \otimes C}$ (Theorem 2.3). Then we let $C=H$ and $M=I$, the identity object of $\mathcal{C}$, and present an analogous of Theorem 7.1 in [9], but again with the symmetric condition for $\mathcal{C}$ replaced, in this case, by only one restriction on $\psi_{H} \otimes H$ (Theorem 2.4). The latter case is the braided version of Connes-Moscovici's Hopf cyclic cohomology in a non-symmetric category $\mathcal{C}$ in which only the relation $\psi_{H \otimes H}^{2}=i d_{H \otimes H}$ holds.

Let us first recall two definitions [9]. They are needed for the following theorems. Let $\mathcal{C}$ be a braided monoidal category and $(H, m, \eta, \Delta, \varepsilon, S)$ a Hopf algebra object (braided Hopf algebra) in $\mathcal{C}$.

Definition 2.1. A right-left braided stable anti-Yetter-Drinfeld (BSAYD) $H$-module in $\mathcal{C}$ is an object $M$ in $\mathcal{C}$ such that:

(i) $M$ is a right $H$-module via an action $\phi_{M}: M \otimes H \rightarrow M$,

(ii) $M$ is a left $H$-comodule via a coaction $\rho_{M}: M \rightarrow H \otimes M$,

(iii) $M$ satisfies the braided anti-Yetter-Drinfeld condition,

$$
\begin{aligned}
(\rho)(\phi)= & {[(m)(S \otimes m) \otimes \phi]\left[\left(\psi_{H^{\otimes 2}, H} \otimes i d_{M} \otimes i d_{H}\right)\left(i d_{H^{\otimes 2}} \otimes \psi_{M, H} \otimes i d_{H}\right)\right.} \\
& \left.\left(i d_{H^{\otimes 2}} \otimes i d_{M} \otimes \psi_{H, H}\right)\left(i d_{H} \otimes \psi_{M, H} \otimes i d_{H^{\otimes 2}}\right)\right]\left[\rho \otimes \Delta^{2}\right] .
\end{aligned}
$$

(iv) $M$ is stable, i.e., $(\phi)\left(\psi_{H, M}\right)(\rho)=i d_{M}$.

Definition 2.2. A quadruple $\left(C, \Delta_{C}, \varepsilon_{C}, \phi_{C}\right)$ in $\mathcal{C}$ is called a left (braided) $H$-module-coalgebra in $\mathcal{C}$ if $\left(C, \Delta_{C}, \varepsilon_{C}\right)$ is a coalgebra in $\mathcal{C}$, and $C$ is a left $H$-module via an action $\phi_{C}: H \otimes C \rightarrow C$ such that $\phi_{C}$ is a coalgebra map in $\mathcal{C}$, i.e., we have:

$$
\Delta_{C} \phi_{C}=\left(\phi_{C} \otimes \phi_{C}\right)\left(i d_{H} \otimes \psi_{H, C} \otimes i d_{C}\right)\left(\Delta_{H} \otimes \Delta_{C}\right), \quad \varepsilon_{C} \phi_{C}=\varepsilon_{H} \otimes \varepsilon_{C},
$$

Now we state main theorems of this section.

Theorem 2.3. Let $\mathcal{C}$ be an abelian braided monoidal category which is not necessarily symmetric. Consider the triple $(H, C, M)$, where $H$ is a Hopf algebra, $C$ is an $H$-module coalgebra, and $M$ is a right-lef stable anti Yetter-Drinfeld (BSAYD) $H$-module, all in $\mathcal{C}$, for which the following relations hold,

$$
\begin{gathered}
\psi_{H \otimes M} \psi_{M \otimes H}=i d_{M \otimes H}, \quad \psi_{H \otimes C} \psi_{C \otimes H}=i d_{C \otimes H}, \quad \psi_{M \otimes C} \psi_{C \otimes M}=i d_{C \otimes M}, \\
\psi_{C \otimes C} \psi_{C \otimes C}=i d_{C \otimes C}, \quad \psi_{H \otimes H} \psi_{H \otimes H}=i d_{H \otimes H} .
\end{gathered}
$$

Then $\left(C_{H}^{\bullet}, \widetilde{\delta_{i}}, \widetilde{\sigma_{i}}, \widetilde{\tau_{n}}\right)$, defined as follows, is a cocyclic object in $\mathcal{C}$.

First, for $n \geq 0$, we let

$$
C^{n}=C^{n}(C, M):=M \otimes C^{\otimes n+1},
$$

and we define, for $0 \leq i \leq n$, faces $\delta_{i}: C^{\otimes n-1} \rightarrow C^{\otimes n}$ and degeneracies $\sigma_{i}: C^{\otimes n+1} \rightarrow C^{\otimes n}$ and cyclic maps $\tau_{n}: C^{\otimes n} \rightarrow C^{\otimes n}$ by:

$$
\begin{aligned}
& \delta_{i}=i d_{M} \otimes i d_{C \otimes i} \otimes \Delta_{C} \otimes i d_{C \otimes n-i-1}, \quad 0 \leq i<n, \\
& \delta_{n}=\left(i d_{M} \otimes \psi_{C \otimes C \otimes n}\right)\left(i d_{M} \otimes \phi_{C} \otimes i d_{C \otimes n}\right)\left(\psi_{H \otimes M} \otimes i d_{C \otimes n+1}\right)\left(\rho_{M} \otimes \Delta_{C} \otimes i d_{C \otimes n-1}\right), \\
& \sigma_{i}=i d_{M} \otimes i d_{C \otimes i+1} \otimes \varepsilon_{C} \otimes i d_{C \otimes n-i}, \quad 0 \leq i \leq n, \\
& \tau_{n}=\left(i d_{M} \otimes \psi_{C \otimes C \otimes n}\right)\left(i d_{M} \otimes \phi_{C} \otimes i d_{C \otimes n}\right)\left(\psi_{H \otimes M} \otimes i d_{C \otimes n+1}\right)\left(\rho_{M} \otimes i d_{C \otimes n+1}\right) .
\end{aligned}
$$

Next, we form the balanced tensor products (Here is when we need $\mathcal{C}$ to be abelian),

$$
C_{H}^{n}=C_{H}^{n}(C, M):=M \otimes_{H} C^{\otimes n+1}, \quad n \geq 0,
$$

with induced faces, degeneracies, and cyclic maps denoted by $\widetilde{\delta_{i}}, \widetilde{\sigma_{i}}$ and $\widetilde{\tau_{n}}$. 
Proof. As in the proof of Theorem 3.6 in [9], with slight modifications.

Now if we put $M=I$, the identity object of $\mathcal{C}$, and $C=H$ then Theorem 2.3 reduces to the braided version of ConnesMoscovici's Hopf cyclic theory [2, 3, 4], in any monoidal category (not necessarily symmetric), as follows.

Theorem 2.4. Let $\mathcal{C}$ be any abelian braided monoidal category, which is not necessarily symmetric. Let $(H, m, \eta, \Delta, \varepsilon, S)$ be a braided Hopf algebra in $\mathcal{C}$ for which, $\psi_{H \otimes H} \psi_{H \otimes H}=i d_{H \otimes H}$ (for short we show this by $\left.\psi^{2}=i d\right)$. Let $H$ be endowed with a braided modular pair in involution $(B M P I)(\delta, \sigma)$. Then the following data defines a cocyclic object in $\mathcal{C}$ :

$$
\begin{aligned}
& C^{0}(H)=I, \quad \text { and } \quad C^{n}(H)=H^{\otimes n}, \quad n \geq 1, \\
& \delta_{i}= \begin{cases}\eta \otimes i d_{H \otimes n} & i=0 \\
i d_{H^{\otimes i-1}} \otimes \Delta \otimes i d_{H^{\otimes n-i}} & 1 \leq i \leq n-1 \\
i d_{H \otimes n} \otimes \sigma & i=n\end{cases} \\
& \sigma_{i}=i d_{H^{\otimes i-1}} \otimes \varepsilon \otimes i d_{H^{\otimes n-i}}, \quad 0 \leq i \leq n \\
& \tau_{n}= \begin{cases}i d_{I} & n=0 \\
\left(m_{n}\right)\left(\Delta^{n-1} \widetilde{S} \otimes i d_{H \otimes n-1} \otimes \sigma\right) & n \neq 0\end{cases}
\end{aligned}
$$

Here $\widetilde{S}:=(\delta \otimes S) \Delta$ and by $m_{n}$ we mean, $m_{1}:=m$, and for $n \geq 2$ :

$$
m_{n}:=m_{H \otimes n}=(\underbrace{m \otimes m \otimes \ldots \otimes m}_{n \text { times }}) \mathcal{F}_{n}(\psi),
$$

where

$$
\mathcal{F}_{n}(\psi):=\prod_{j=1}^{n-1}(i d_{H^{\otimes j}} \otimes \underbrace{\psi \otimes \psi \otimes \ldots \otimes \psi}_{n-j \text { times }} \otimes i d_{H^{\otimes j}}) .
$$

Proof. As in the proof of Theorem 7.1 in [9], with slight modifications.

We will denote the braided Hopf cyclic cohomology of a braided Hopf algebra, $H$, in Theorem 2.4 by $B H C^{*}(H)$, as we denote the usual Hopf cyclic cohomology of a Hopf algebra, $H$, by $H C^{*}(H)$.

\section{Existence of Hopf algebras with property $\psi^{2}=i d$ in non-symmetric categories}

In this section, first, we show that there are families of non-symmetric categories, namely the category of anyonic vector spaces $[12,14]$, in which for many objects $A$ and $B$ the property $\psi_{B \otimes A} \psi_{A \otimes B}=i d_{A \otimes B}$ holds (for $A=B$ this means $\left.\psi_{A \otimes A}^{2}=i d_{A \otimes A}\right)$. Next, within one set of those non-symmetric categories, we provide families of examples of Hopf algebra objects. These are anyonic Hopf algebras, H, for which $\psi_{H \otimes H}^{2}=i d_{H \otimes H}$. Therefore, our Theorem 2.4 could be applied to them. In the next sections we set out to compute the Hopf cyclic cohomology of these Hopf algebras.

We need to recall that $[1,12,14]$, if $\left(H, R=R_{1} \otimes R_{2}\right)$ is a quasitriangular Hopf algebra and $\mathcal{C}$ the category of all left $H$-modules, then $\mathcal{C}$ is a braided monoidal abelian category. Here the monoidal structure is defined by

$$
h \triangleright(v \otimes w)=h^{(1)} \triangleright v \otimes h^{(2)} \triangleright w,
$$

where $\triangleright$ denotes the action of $H$, and the braiding map, $\psi$, is defined by

$$
\psi_{V \otimes W}(v \otimes w):=\left(R_{2} \triangleright w \otimes R_{1} \triangleright v\right)
$$

for any $V$ and $W$ in $\mathcal{C}$.

Now let $H=\mathbb{C Z}_{n}$, the group (Hopf) algebra of the finite cyclic group, $\mathbb{Z}_{n}$, of order $n$. In addition to the trivial $R=1 \otimes 1$ there exists a nontrivial quasitriangular structure for $H=\mathbb{C Z}_{n}$, defined by [14]:

$$
R=(1 / n) \sum_{a, b=0}^{n-1} e^{\frac{(-2 \pi i a b)}{n}} g^{a} \otimes g^{b}
$$

where $g$ is the generator of $\mathbb{Z}_{n}$. The category of all left $H$-modules, denoted here by $\mathcal{C}$, is known as the category of anyonic vector spaces. The objects of $\mathcal{C}$ are of the form $V=\bigoplus_{i=0}^{n-1} V_{i}$. They are $\mathbb{Z}_{n}$-graded representations of $\mathbb{C Z}_{n}$ and the action of $\mathbb{Z}_{n}$ on $V$ is given by,

$$
g \triangleright v=e^{\frac{2 \pi i|v|}{n}} v,
$$

where $|v|=k$ is the degree of the homogeneous elements $v$ in $V_{k}$. The morphisms of $\mathcal{C}$ are linear maps that preserve the grading. Applying formulas (2) and (3) to (1) will give us the formula for the braiding map in $\mathcal{C}$ as:

$$
\psi_{V \otimes W}(v \otimes w)=e^{\frac{2 \pi i|v||w|}{n}} w \otimes v,
$$


where $|v|$ and $|w|$ are the degrees of homogeneous elements $v$ and $w$ in objects $V$ and $W$, respectively.

This category is not symmetric when $n>2$. But, in the following examples, we will prove that for many values of $n$ one can always find objects $A$ and $B$ in $\mathcal{C}$ such that, $\psi_{B \otimes A} \psi_{A \otimes B}=i d_{A \otimes B}$. Here is one set of examples:

Lemma 3.1. Let $\mathcal{C}$ be the category of left $\mathbb{C Z}_{n}$-modules, where $n=2 m^{2}$ for some integer $m>1$. Let $A=\bigoplus_{i=0}^{n-1} A_{i}$ and $B=\bigoplus_{i=0}^{n-1} B_{i}$ be objects in $\mathcal{C}$ which are focused only in degrees, $k m$, for integers $k \geq 0$. For any two homogeneous elements $x$ in $A$ and $y$ in $B$, with $|x|=k m$ and $|y|=l m, k, l \geq 0$, by Formula (4), we have,

$$
\psi_{A \otimes B}(x \otimes y)=e^{\frac{2 \pi i|x||y|}{2 m^{2}}}(y \otimes x)=(-1)^{\frac{|x||y|}{m^{2}}}(y \otimes x)=(-1)^{k l}(y \otimes x) .
$$

Thus, it is clear that,

$$
\psi_{B \otimes A} \psi_{A \otimes B}=i d_{A \otimes B}
$$

By an object, $A$, being focused only in degrees $k m$ we mean, $A_{i}=0$ when $i \neq k m$, for integers $k \geq 0$. An example of the above Lemma is the case when $n=18$ and $A=\bigoplus_{i=0}^{17} A_{i}$ and $B=\bigoplus_{i=0}^{17} B_{i}$ are objects focused only in degrees 0,3 , $6,9,12$, and 15 .

Remark 3.2. Formula (5) shows that even though the objects in Lemma 3.1, A and B, live in a truly braided category, their corresponding braid maps, $\psi_{A \otimes B}$ and $\psi_{B \otimes A}$, behave almost like the braid maps for objects in the category of super vector spaces, by replacing $|x|$ and $|y|$ in the super case with $\frac{|x|}{m}$ and $\frac{|y|}{m}$.

Now we define a family of Hopf algebra objects, within the objects of Lemma 3.1. For such an anyonic Hopf algebra $[12,14], H$, Formula (6) translates to $\psi_{H \otimes H}^{2}=i d_{H \otimes H}$, as desired.

Lemma 3.3. Let $\mathcal{C}$ be the category of left $\mathbb{C Z}_{n}$-modules, where $n=2 m^{2}$ for some integer $m>1$. We define a Hopf algebra object, $H=\bigoplus_{i=0}^{n-1} H_{i}$, in $\mathcal{C}$, focused in degrees $k m$, for integers $k \geq 0$, as follows. As an algebra, we define $H$ to be generated by elements $x_{i}$ in degrees $i m$ (i.e., $\left|x_{i}\right|=i m$ ), for $i \geq 0$, subject to no relations (except, of course, $x_{i}^{0}=1$ ). We define the rest of the Hopf algebra structure on $H$ by,

$$
\Delta\left(x_{i}\right)=1 \otimes x_{i}+x_{i} \otimes 1, \quad \epsilon\left(x_{i}\right)=0, \quad S\left(x_{i}\right)=-x_{i}
$$

and extend $\Delta$ and $\epsilon$ as (braided) algebra maps, and $S$ as an anti-commutative algebra map. This means (by Formula (5)), for any homogeneous elements $v$ and $w$ in $H$,

$$
\Delta(v w)=(-1)^{\frac{\left|v^{(2)}\right|\left|w^{(1)}\right|}{m^{2}}} v^{(1)} w^{(1)} \otimes v^{(2)} w^{(2)}, \quad \epsilon(v w)=\epsilon(v) \epsilon(w)
$$

and $S(v w)=(-1)^{\frac{|v||w|}{m^{2}}} S(w) S(v)$. These imply that, for example, if $\left|x_{i}\right|=i m$ and $\left|x_{j}\right|=j m$ are both odd then we have,

$$
\Delta\left(x_{i} x_{j}\right)=1 \otimes x_{i} x_{j}-x_{j} \otimes x_{i}+x_{i} \otimes x_{j}+x_{i} x_{j} \otimes 1, \quad \epsilon\left(x_{i} x_{j}\right)=0, S\left(x_{i} x_{j}\right)=-x_{j} x_{i},
$$

and if one of $\left|x_{i}\right|$ or $\left|x_{j}\right|$ is even,

$$
\Delta\left(x_{i} x_{j}\right)=1 \otimes x_{i} x_{j}+x_{j} \otimes x_{i}+x_{i} \otimes x_{j}+x_{i} x_{j} \otimes 1, \quad \epsilon\left(x_{i} x_{j}\right)=0, \quad S\left(x_{i} x_{j}\right)=x_{j} x_{i} .
$$

In fact, in Lemma 3.3, there is no need to restrict ourselves to one generator for each degree. This means we can have, for example, a set of generators, $\left\{x_{i 1}, x_{i 2}, x_{i 3}, \cdots\right\}$, in each degree $i m$.

The structure defined in Lemma 3.3 is too general. Below, we provide some simpler examples by working with an odd integer $m$, limiting the number of generators, and by imposing some relations into the algebra structure. They are easier, for our purposes, to work with and we will use them in the next sections. In particular, we consider the case $n=18$, i.e., $m=3$, very often.

Example 3.4. Let $\mathcal{C}$ be the category of left $\mathbb{C Z}_{n}$-modules, where $n=2 m^{2}$ for some odd integer $m>1$. We define a Hopf algebra object $H=\bigoplus_{i=0}^{n-1} H_{i}$, in $\mathcal{C}$, as follows. As an algebra, we define $H$ to be generated by elements $x_{i}$ in degrees im, for $i=1,3,5,7, \cdots, 2 m-1$, subject to the following relations: For $i, j=1,3,5,7, \cdots, 2 m-1, x_{i}^{0}=1, \quad x_{i} x_{j}=-x_{j} x_{i}$. We define the rest of Hopf algebra structure on $H$ by, $\Delta\left(x_{i}\right)=1 \otimes x_{i}+x_{i} \otimes 1, \quad \epsilon\left(x_{i}\right)=0$, and $S\left(x_{i}\right)=-x_{i}$, and extend $\Delta$ and $\epsilon$ as (braided) algebra maps and $S$ as an anti-commutative algebra map.

The following examples are yet simpler versions of the above ones, obtained by adding the relation $x_{i}^{2}=0$.

Example 3.5. Let $\mathcal{C}$ be the category of left $\mathbb{C Z}_{n}$-modules, where $n=2 m^{2}$ for some odd integer $m>1$. We define a Hopf algebra object, $H=\bigoplus_{i=0}^{n-1} H_{i}$, in $\mathcal{C}$, as follows. As an algebra, we define $H$ to be generated by elements $x_{i}$ in degrees $i m$, for $i=1,3,5,7, \cdots, 2 m-1$, subject to the relations: $x_{i}^{2}=0, \quad x_{i}^{0}=1, \quad x_{i} x_{j}=-x_{j} x_{i}$, for $i, j=1,3,5,7, \cdots, 2 m-1$. The rest of Hopf algebra structure on $H$ is defined by, $\Delta\left(x_{i}\right)=1 \otimes x_{i}+x_{i} \otimes 1, \quad \epsilon\left(x_{i}\right)=0$, and $S\left(x_{i}\right)=-x_{i}$, with $\Delta$ and $\epsilon$ extended as (braided) algebra maps and $S$ as an anti-commutative algebra map.

In particular if $n=18$, we have,

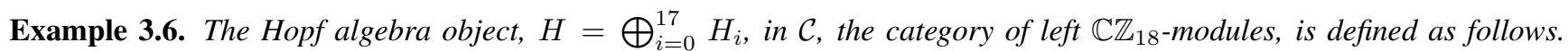
As an algebra, $H$ is generated by elements $x, y$, and $z$, of degrees 3,9 , and 15 , respectively, subject to the relations, $x^{2}=y^{2}=z^{2}=0, \quad x^{0}=y^{0}=z^{0}=1, \quad x y=-y x, \quad x z=-z x$, $y z=-z y$. Regarding the rest of Hopf algebra structure on $H$, for $v=x, y, z, \Delta(v)=1 \otimes v+v \otimes 1, \quad \epsilon(v)=0, \quad S(v)=$ $-v$, with $\Delta$ and $\epsilon$ extended as (braided) algebra maps and $S$ as an anti-commutative algebra map. 


\section{Some quantum Lie algebras and their enveloping Hopf algebras}

We follow [6] for the definitions and related notions that we are going to use in what follows.

In this section we define a few particular examples of quantum Lie algebras, $\mathfrak{g}$, (in the sense of [6]), for which their universal enveloping algebras, $U(\mathfrak{g})$, turn out to be the anyonic Hopf algebras of Section 3. This fact not only is interesting on its own, but also will play an important role in Section 5 where we want to compute the braided Hopf cyclic cohomology of those anyonic Hopf algebras.

Lemma 4.1. Let $\mathcal{C}$ be the category of left $\mathbb{C Z}_{n}$-modules, where $n=2 m^{2}$, for some odd integer $m>1$. We define an object, $\mathfrak{g}=\bigoplus_{i=0}^{n-1} \mathfrak{g}_{i}$, in $\mathcal{C}$, by setting $\mathfrak{g}_{i m}=\mathbb{C} x_{i}$ and $\mathfrak{g}_{2 i m}=\mathbb{C} a_{i}$, for $i=1,3,5,7, \cdots, 2 m-1$, and $\mathfrak{g}_{i}=0$, otherwise. We define a Lie bracket on $\mathfrak{g}$ by $\left[x_{i}, x_{i}\right]=a_{i}$, for $i=1,3,5,7, \cdots, 2 m-1$, and $[]=$,0 otherwise. Then, $\left(\mathfrak{g}, \psi_{\mathfrak{g} \otimes \mathfrak{g}},[],\right)$ is a left quantum Lie algebra, in the sense of Definition 2.1 in [6].

Proof. Since $\psi_{\mathfrak{g} \otimes \mathfrak{g}}(v \otimes w)=(-1)^{\frac{|v||w|}{m^{2}}} w \otimes v$, properties (1) and (3) in Definition 2.1 in [6] are automatically satisfied. Also, since the only elements in $\operatorname{ker}(i d-\psi)$ are $a_{i} \otimes a_{i}$, and since by our definition $\left[a_{i}, a_{i}\right]=0$, property (4) is satisfied too. All one needs to check is property (2), the quantum Jacobi identity. On one hand, if one of the components in any bracket $[*, *]$ is $a_{i}$, then $[*, *]=0$. On the other hand, $[*, *]$ is equal to either zero or $a_{i}$. These two statements imply that any bracket of the form $[*,[*, *]]$ or $[[*, *], *]$ (appearing in (2)) is equal to zero. Therefore, in (2), we always get both sides equal to zero, and we are done.

By Lemma 2.6 in [6], for the quantum Lie algebra, $\mathfrak{g}$, in the above example, the universal enveloping algebra, $U(\mathfrak{g})$, is a Hopf algebra [12,14] with the standard structure. Let us see what this Hopf algebra is. By the definition of $U(\mathfrak{g})[6]$ and Formula (5), we have,

$$
U(\mathfrak{g})=\frac{T(\mathfrak{g})}{\left(v \otimes w-(-1)^{\frac{|v||w|}{m^{2}}} w \otimes v-[v, w]\right)} .
$$

This means that in $U(\mathfrak{g}),[v, w]=v w-w v$, when $|v||w|$ is an even multiple of $m^{2}$, and $[v, w]=v w+w v$, when $|v||w|$ is an odd multiple of $m^{2}$. When we apply these to the basis $x_{i}$, and $a_{i}$, we get, for $i, j=1,3,5,7, \cdots, 2 m-1$,

$$
a_{i}=\left[x_{i}, x_{i}\right]=2 x_{i}^{2}, \quad 0=\left[x_{i}, x_{j}\right]=x_{i} x_{j}+x_{j} x_{i}, i \neq j .
$$

Therefore, $U(\mathfrak{g})$ for the $\mathfrak{g}$ of Lemma 4.1, is, in fact, the anyonic Hopf algebra of Example 3.4.

Now in the same manner, we recover the remaining Hopf algebras in Section 3 as enveloping algebras of some (abelian) quantum Lie algebras.

Lemma 4.2. Let $\mathcal{C}$ be the category of left $\mathbb{C Z}_{n}$-modules, where $n=2 m^{2}$, for some odd integer $m>1$. We define $\mathfrak{g}=$ $\bigoplus_{i=0}^{n} \mathfrak{g}_{i}$, in $\mathcal{C}$, by setting, $\mathfrak{g}_{i m}=\mathbb{C} x_{i}$, for $i=1,3,5,7, \cdots, 2 m-1$, and $\mathfrak{g}_{i}=0$, otherwise. We define the Lie bracket on $\mathfrak{g}$, to be zero. Then, it is very easy to see that $\left(\mathfrak{g}, \psi_{\mathfrak{g} \otimes \mathfrak{g}},[],\right)$ is a quantum abelian Lie algebra.

For $\mathfrak{g}$ of this Lemma, in a similar manner as before, and with easier calculations (as $\mathfrak{g}$ is abelian), we will see that $U(\mathfrak{g})$ is the anyonic Hopf algebra of Example 3.5.

When we let $m=3$ in the above Lemma, we have,

Example 4.3. Let $\mathfrak{g}=\bigoplus_{i=0}^{17} \mathfrak{g}_{i}$, as an object in $\mathcal{C}$, the category $\mathcal{C}$ of left $\mathbb{C} \mathbb{Z}_{18}$-modules, be defined by setting $\mathfrak{g}_{i}=\mathbb{C} x_{i}$, for $i=1,3,5$ (note, $\left|x_{i}\right|=3 i$ ), and $\mathfrak{g}_{i}=0$, otherwise. We define the Lie bracket on $\mathfrak{g}$, to be zero. The enveloping algebra $U(\mathfrak{g})$, for this quantum abelian Lie algebra, is the anyonic Hopf algebra of Example 3.6.

In Section 5 we will use the results of this section, Theorem 2.4 from Section 2, and an idea similar to the one used for super Hopf algebras in [9], to compute the braided periodic Hopf cyclic cohomology of $H=U(\mathfrak{g})$ from the above examples.

\section{Computing braided Hopf cyclic cohomology using Theorem 2.4}

In this section we want to apply the result in Theorem 2.4 to the anyonic Hopf algebras from Section 3, and compute their (braided) periodic Hopf cyclic cohomology. In Section 4, we proved that for any of those Hopf algebras $H$, we have $H=U(\mathfrak{g})$, for some quantum Lie algebra, $\mathfrak{g}$.

This is why the main result of this section is the following theorem. It is an analogue of Theorem 6.3 in [9], adapted for quantum Lie algebras in the category of anyonic vector spaces, within the family of Lemma 3.1.

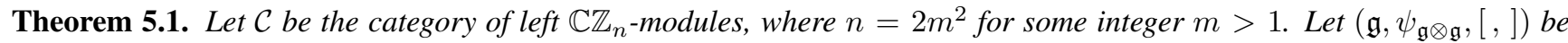
a quantum Lie algebra in $\mathcal{C}$, focused only in degrees, $k m$, for integers $k \geq 0$ (i.e., within the family of Lemma 3.1). Then, analogous to the non-super case [2, 3, 4, 5] and the super case [9], the relation,

$$
H P_{(\delta, 1)}^{*}(U(\mathfrak{g}))=\bigoplus_{i=*(\bmod 2)} H_{i}\left(\mathfrak{g} ; \mathbb{C}_{\delta}\right), \quad *=0,1,
$$


holds, where $\delta$ is a character for $\mathfrak{g}$. Here, $H P_{(\delta, 1)}^{*}(U(\mathfrak{g}))$ is the periodic Hopf cyclic cohomology of the anyonic Hopf algebra $H=U(\mathfrak{g})$, and $H_{i}\left(\mathfrak{g} ; \mathbb{C}_{\delta}\right)$ is the Lie algebra homology of $\mathfrak{g}$, with coefficients in the $\mathfrak{g}$-module $\mathbb{C}_{\delta}$.

Proof. For the reason mentioned in Remark 3.2, it is very straightforward to see that the proof of this theorem is similar to the one of Theorem 6.3 in [9], given in detail therein. All one needs to do is to replace all the degrees of the homogeneous elements in the proof of that theorem, such that a given degree, $|x|$, becomes $\frac{|x|}{m}$.

The Lie algebra homologies, $H_{i}\left(\mathfrak{g} ; \mathbb{C}_{\delta}\right)$, in the above theorem, are computed by the following complex, which is an analogue of the Chevalley-Eilenberg complex, adapted for $\mathfrak{g}$ within the family of Example 3.1.

$$
\begin{aligned}
& \bigwedge^{0} \mathfrak{g}<\delta \\
\bigwedge^{1} \mathfrak{g}<d & \bigwedge^{2} \mathfrak{g}<d \\
d\left(x_{1} \wedge \ldots \wedge x_{i}\right) & =\sum_{k=1}^{i}(-1)^{k+1+\alpha_{k}} \delta\left(x_{k}\right)\left(x_{1} \wedge \ldots \wedge \widehat{x}_{k} \wedge \ldots \wedge x_{i}\right) \\
& +\sum_{1 \leq k<l \leq i}(-1)^{k+l+\beta_{k, l}}\left(\left[x_{k}, x_{l}\right] \wedge x_{1} \wedge \ldots \wedge \widehat{x}_{k} \wedge \ldots \wedge \widehat{x}_{l} \wedge \ldots \wedge x_{i}\right),
\end{aligned}
$$

where, $\alpha_{1}:=0, \alpha_{k}:=\frac{\left|x_{k}\right|\left(\left|x_{1}\right|+\ldots+\left|x_{k-1}\right|\right)}{m^{2}}$ for $k>1, \beta_{k, l}:=\alpha_{k}+\alpha_{l}-\frac{\left|x_{k}\right|\left|x_{l}\right|}{m^{2}}$ for $k<l$, and $\widehat{x}_{k}$ means omitted. Also by definition,

$$
\bigwedge(\mathfrak{g})=\bigoplus_{i=0}^{\infty} \bigwedge^{i} \mathfrak{g}:=\frac{T(\mathfrak{g})}{\left(a \otimes b+(-1)^{\frac{|a||b|}{m^{2}}} b \otimes a\right)} .
$$

Now, one can apply this theorem to any of the anyonic Hopf algebras in Section 3. As an example, we close this paper by applying Theorem 5.1, in detail, to the anyonic Hopf algebra of Example 3.6, proved to be the enveloping algebra of the quantum Lie algebra of Example 4.3.

In Formula (9), since $\delta=\varepsilon$, and the Lie algebra brackets are zero, all the boundary maps are zero. Thus $H_{i}\left(\mathfrak{g}, \mathbb{C}_{\varepsilon}\right)=$ $\bigwedge^{i} \mathfrak{g}$, where by Formula (10), $\bigwedge^{i} \mathfrak{g}$, as a vector space, is generated by all elements of the form $\wedge^{k} x \wedge \wedge^{l} y \wedge \wedge^{p} z$ such that $k+l+p=i$. Here, by $\wedge^{k} x$ we mean $x \wedge x \wedge x \cdots \wedge x, k$ times.

On the other hand, since in $\mathfrak{g}=\bigoplus_{i=0}^{17} \mathfrak{g}_{i},|x|=3,|y|=9$, and $|z|=15$, we have $\left|\wedge^{k} x \wedge \wedge^{l} y \wedge \wedge^{p} z\right|=3 k+9 l+15 p=$ $3(i+(2 l+4 p)),(\bmod 18)$. This implies that, as an anyonic vector space, $H_{i}(\mathfrak{g})=\bigoplus_{t=0}^{17} H_{i}(\mathfrak{g})_{t}$ is focused only in degrees that are even multiples of 3 when $i$ is even, and in degrees that are odd multiples of 3 when $i$ is odd.

Therefore by Formula $(8), H P_{(\varepsilon, 1)}^{0}(U(\mathfrak{g}))=\bigoplus_{i=0(\bmod 2)} \bigwedge^{i} \mathfrak{g}$, as an anyonic vector space, is focused in degrees that are even multiples of 3 , with infinitely many elements in the basis of each degree. Similarly, as an anyonic vector space, $H P_{(\varepsilon, 1)}^{1}(U(\mathfrak{g}))=\bigoplus_{i=1(\bmod 2)} \wedge^{i} \mathfrak{g}$ is focused in degrees that are odd multiples of 3 , with infinitely many elements in the basis of each degree.

\section{REFERENCES}

[1] D. Bulacu, and E. Nauwelaerts, Radford's biproduct for quasi-Hopf algebras and bosonization, J. Pure Appl. Algebra 174 (2002), no. $1,1-42$.

[2] A. Connes, and H. Moscovici, Hopf algebras, cyclic cohomology and the transverse index theorem, Comm. Math. Phys. 198 (1998), no. 1, 199-246.

[3] A. Connes, and H. Moscovici, Cyclic cohomology and Hopf algebras, Lett. Math. Phys. 48 (1999), no. 1, 97-108.

[4] A. Connes, and H. Moscovici, Cyclic cohomology and Hopf algebra symmetry, Conference Mosh Flato 1999 (Dijon), Lett. Math. Phys. 52 (2000), no. 1, 1-28.

[5] M. Crainic, Cyclic cohomology of Hopf algebras. J. Pure Appl. Algebra 166 (2002), no. 1-2, 29-66.

[6] X. Gomez, and S. Majid, Braided Lie algebras and bicovariant differential calculi over co-quasitriangular Hopf algebras, Journal of Algebra 261 (2003), 334-388.

[7] P. M. Hajac, M. Khalkhali, B. Rangipour, and Y. Sommerhäuser, Stable anti-Yetter-Drinfeld modules, C. R. Math. Acad. Sci. Paris 338 (2004), no. 8, 587-590.

[8] P. M. Hajac, M. Khalkhali, B. Rangipour, and Y. Sommerhäuser, Hopf-cyclic homology and cohomology with coefficients, C. R. Math. Acad. Sci. Paris 338 (2004), no. 9, 667-672.

[9] M. Khalkhali, and A. Pourkia, Hopf Cyclic Cohomology in Braided Monoidal Categories, Homology, Homotopy and Applications (HHA), Vol. 12 (2010), no. 1, 111-155.

[10] J. L. Loday, Cyclic homology, Second edition, Grundlehren der Mathematischen Wissenschaften [Fundamental Principles of Mathematical Sciences], 301, Springer-Verlag, Berlin, 1998. 
[11] S. Mac Lane, Categories for the working mathematician, Second edition, Graduate Texts in Mathematics, 5, Springer-Verlag, New York, 1998.

[12] S. Majid, Algebras and Hopf algebras in braided categories, Advances in Hopf algebras, 55-105, Lecture Notes in Pure and Appl. Math. 158, Dekker, New York, 1994.

[13] S. Majid, Quantum and braided Lie algebras, J. Geom. Phys. 13, no. 4, (1994), 307-356.

[14] S. Majid, Foundations of quantum group theory, Cambridge University Press, Cambridge, 1995.

[15] A. Pourkia, A survey of braided Hopf cyclic cohomology, Fields Institute Communications, (Perspectives on Noncommutative Geometry) Vol. 61 (2011), 117-131.

[16] S.L.Woronowicz, Differential calculus on compact matrix pseudogroups (quantum groups), Comm. Math. Phys. 122 (1989), 125-170. 